Note: This is a draft of a paper being submitted for publication. Contents of this paper should not be quoted nor referred to without permission of the authors.

BACKSCATTERING OF LIGHT IONS FROM METAL SURFACES

H. Verbeek

By acceptance of this article, the publisher or recipient acknowledges the U. S. Government's right to retain nonexclusive, royalty-free license in and to any copyright covering the article.

SOLID STATE DIVISION

OAK RIDGE NATIONAL LABORATORY

Operated by

UNION CARBIDE CORPORATION

for the

ENERGY RESEARCH AND DEVELOPMENT ADMINISTRATION

Oak Ridge, Tennessee 


\title{
BACKSCATTERING OF LIGHT IONS FROM METAL SURFACES*
}

\author{
H. Verbeekt \\ Solid State Division \\ Oak Ridge National Laboratory \\ Oak Ridge, Tennessee, 37830
}

\begin{abstract}
When a metal target is bombarded with light ions some are implanted and some are reflected from the surface or backscattered from deeper layers. This results in an energy distribution of the backscattered particles which reaches from zero to almost the primary energy. The number of the backscattered particles and their energy, angular, and charge distributions depends largely on the energy and the ion target combination. For high energies (i.e. $>50 \mathrm{keV}$ for protons) particles are backscattered in a single collision governed by the Rutherford cross section. Protons and He-ions with energies of $100 \mathrm{keV}$ to several MeV are widely used for thin film analysis. For lower energies multiple collisions and the screening of the coulomb potential have to be taken into account, which makes the theoretical treatment more difficult. This energy region is, however, of special interest in the field of nuclear fusion research. Some recent results for energies below $20 \mathrm{keV}$ are discussed in some detail.
\end{abstract}

\footnotetext{
iResearch sponsored by the U. S. Energy Research and Development Administration under contract with the Union Carbide Corporation.

TGuest Scienti.st from the Max P.lanck Institut für Plasmaphysik, 0-8046 Garching. West Germany.
} 


\section{INTRODUCTION}

When a beam of jons impinges onto a metal target, some of the ions are implanted and some are reflected from the surface or backscattered from deeper layers. The implanted atoms may diffuse to the surface and be released with thermal energies or they may be trapped inside the material, e.g. at lattice defects. They also may cluster together and form gas bubbles inside the metal. The amount of trapping depends strongly on the particular ion target combination, on the temperature, and on the bombardment dose. This paper deals only with the particles which are kinetically backscattered, and is restricted to light lons such as hydrogen and helium.

The particles which are backscattered give rise to an energy distribution which extends from zero energy to almost the primary energy. A typical example is given in Fig. 1, which shows the energy distribution for $15 \mathrm{keV}$ protons backscattered from an Au target. The sharp threshold at high energies is due to ions which are backscattered from the surface atoms of the target. The position of this edge is determined by the kinematics of a single scattering event. When a particle of mass $M_{2}$ and an energy $E_{2}$ is scattered from an atom with mass $M_{2}$ the energy after scattering is

$$
E_{2}=k^{2} E_{1}
$$

where $\quad k=\left[M_{1} \cos \theta+\left(M_{2}^{2}-M_{1}^{2} \sin ^{2} \theta\right)^{\frac{1}{2}}\right] /\left(M_{1}+M_{2}\right)$

for $M_{1}<M_{2}$ and $\theta$ is the scattering angle in the laboratory system.

The backscattering intensity in Fig. 1 at lower energies results from scattering events deeper in the solid. Along their passage through the solid the particles lose energy in elastic nuclear collisions and quasicontinuously by excitation of target electrons $(1,2)$. Therefore, they 
appear outside the target with all energies below the energy of the particles backscattered from the surface.

This paper is divided into three sections, with each treating a certain range of primary energies. As will be shown these energy ranges arise quite naturally from the different theoretical and experimental treatments of the backscattering effect they requile.

For protons at energles above $\approx 50 \mathrm{keV}$ the scattering can be well described by the single collision model $(3,4,5)$. This model assumes that the backscattering occurs in a single large angle defilection from a certain nuclear collision, which is describable by the Rutherford cross section $(6)$. The trajectorles of the ions inside the material to the scattering center and back to the surface are taken as straight lines. Along their trajectories they lose energy by excltation of the target electrons. This can be described by the differential energy loss dE/dx, i.e. the energy loss per unit path liength. This makes the theoretical calculation of the energy spectra rather simple.

For this energy range the most convenient measuring method ut ilizes surface barrier detectors. These give energy proportional signals which are easily analyzed in a multi-channel analyzer system. They are equally sensitive to ions and neutral atoms.

At high energies, depending strongly on the lon target combination, the validity of the Rutherford scattering law is IImited by the occurrence of nuclear reactions and resonances. This area will be covered by R. S. Blewer, this conference. A compilation of nuclear reaction data may be found In Refs. 7 and 8 . 
Protons and helium lons which are easily available from small accelerators with energies from $\approx 100 \mathrm{keV}$ to several MeV are widely used for surface layer analysis and depth prefiling $(9)$.

At energies below $\approx 50 \mathrm{keV}$ the screening of the nuclear charge by the outer electrons becomes more important. Thus the Coulomb potential leading to the Rutherford cross section is no longer appropriate. More complicated potentials which are derived from the Thomas-fermi theory have to be used ${ }^{(2)}$. Also the validity of the single collision model breaks down. With decreasing energy the cross section for nuclear collisions increases and one has to account for multiple collisions of the backscattered particles. The theoretical treatment becomes much more complicated. An analytical form for the energy distributions can no longer be derived.

Also the experimental techniques must be modified. At energies below * $20 \mathrm{keV}$ surface barrler detectors are no longer suitable to give energy information. Electrostatic or magnetic energy analyzers can be used, but these are only sensitive to charged partlcles. Also, the charged fraction of the backscattered particles decreases with decreasing energy $(10,11,12)$ and the spectra of the neutral and charged components may be very different ${ }^{(11)}$. Thus one needs a means to ionlze the neutral backscattered particles in a definite manner, if quantitative results are to be obtained. Another method to overcome this difflculty is to use time of flight techniques (13), but these too require particle detectors with known sensitivity for neutrals.

The range of primary energies 5 to $20 \mathrm{keV}$ is of special interest for fusion technology. For plasma experiments and later fusion reactors where 
the mean particle confinement times in the plasma are much shorter than the desired burning times, an important role is played by the interaction of the diffusing plasma particles with the first walls. Particularly with respect to the question of recycling ${ }^{(14)}$, it is important to know the total number and angular, energy, and charge distributions of light ions (H, D, T, He) backscattered from soli.d surfaces.

Below the primary energy of $* 1 \mathrm{keV}$ the experiments are extremely difficult. Most of the backscattered partlcles are neutral and the ionization and detection methods currently in use break down at these low energies. To my knowledge there are no experiments -in this energy range dealing with the total number of backscattered particles. Consequently, one has to rely on computer simulations. The backscattering of primary ions with energies below $1 \mathrm{keV}$ are of particular interest for today's plasma experiments.

If one investigates the backscattering from single crystals the results are largely influenced by the crystal structure which gives rise to channeling and blocking effects. This offers a large varlety of measuring . methods in depth profiling and lattlce site determining. . Recent reviews are given in Refs. (15) and (16); the present review deals only with the backscattering from amprphous or polycrystalline materials.

\section{HIGH ENERGIES}

The principle of backscattering of ions with primary energies larger than $* 50 \mathrm{keV}$ can be explained with the aid of Fig. 2. A particle of Energy $E_{1}$ enters a solid at an angle $\alpha$ to the surface normal. The trajectory inside.the solid is a straight line until it encounters a target atom at a distance close enough to cause a large angle defiection. The 
outgoing ion trajectory is again .taken as a stralght line and it leaves the surface at an angle $\beta$.to the normal. (In the experiment $\alpha$ and $\beta$ are determined by the primary beam dl rection and the position of the detector with respect to the target.)

Along their paths through the solid the particles lose energy. For light -ions with energles above -few kel the energy loss is primarily due to ionlzation and excitation of target electrons $(17)$. One can describe that by the differential energy. loss $\mathrm{dE} / \mathrm{dx}$.measured ir. $\circ V / \AA$ or MeV/!(mg/ $\left.\mathrm{cm}^{2}\right)$. Sometimes it i.s more convenlent to use -the stopping cross section or stopping power $\varepsilon=1 / N \mathrm{dE} / \mathrm{dx}=M_{2} /\left(N_{0} p\right) d E / d x$, where $N$ is the number of target atoms per unit volume, $N_{0}$ Avogadro's number, $\rho$ the density and $M_{2}$ the mass number of the target atoms. Thus $\varepsilon$ is measured in $\mathrm{eV} \mathrm{cm}^{2} / \mathrm{atom}$. $\mathrm{dE} / \mathrm{dx}$ (or $\varepsilon$ ) is a function of energy. Today a number of stopping powar tables are available $(18,19,20)$. These are semlampirical tables based on experimental data which are inter- and extrapolated using theoretlcal functional dependences. As an example. Fig. -3.shows dE/dx curves for $\mathrm{H}^{+}$and $\mathrm{He}^{+}$in $\mathrm{NI}$ from the tables of Northcllffe and SchllIIIng (19) A review on the varlous energy loss mechanl.sms has recently been given by Sigmund $(21)$.

With the scheme of Fig. 2 the energy of the outcoming particles $E_{2}$ can be related to the depth from which the backscattering occurs. Particles which are backscattered from the surface have lost energy oniy in the elastic collision, i.e. $E_{2}=k^{2} E_{1}$ according to formula (1). For regions not too far from the surface the relation between depth $x$ and the observed energy $E_{2}$ is: 


$$
\Delta E=k^{2} E_{1}-E_{2}(x)=\left(\frac{k^{2}}{\cos a}\left(\frac{d E}{d x}\right)_{E_{1}}^{-7-}+\frac{1}{\cos B}\left(\frac{d E}{d x}\right)_{E_{2}}\right) x
$$

$\Delta E$ is the difference betwean the energies of particles backscattered from the surface and from a depth $x_{1}(d E / d x)_{E_{1}}$ and $(d E / d x)_{E_{2}}$ are the differentlal energy losses for the mean energies along Incoming and outcoming trajectories. For this formula it was assuned that $d E / d x$ varies only slightiy along the trajectories of the particles. For the analysis of thick layers the target can be divided into thin slices. For each slice the Incident energy can be calculated using the $d E / d x$ surve. In many laboratories, computer programs are used for this procadure.

When composite targets--compounds or mixtures--are investigates, one obtains an overlay of the spectre of each component. These are shifted in energy relative to each other due to the dependarice of $k^{2}$ on the target atom mass number $M_{2}$ (Formula 1 ). The stopping power for a compound is the sum of the stopping powers of the constituents wighted by the relative amounts with wich they occur in the compound (Aregg's rule (22), This relation has been proved valld in a number of cases $(23)$. For instance, the stopping power in $\mathrm{SiO}_{2}$ is $\varepsilon_{\mathrm{SIO}_{2}}-\varepsilon_{\mathrm{Si}}+2 \varepsilon_{0}$. A rigorous treatment of the analysis of targets whose composition varies with depth was given by Brice $(24)$.

An exemple of these principles is presented in fig. 4, which shows the spectrum of $2 \mathrm{MaV} \mathrm{He}$ lons beckscattered from a fll ln of $\mathrm{Mb}_{3} \mathrm{Ge}$ (o compound wi th high superconducting $\mathrm{T}_{\mathrm{c}} \mathrm{j}$ deposited on an $\mathrm{Al}_{2} \mathrm{O}_{3}$ sulstrate ${ }^{(25)}$. The spectro of the constituents of the film and of the substrate are clearly visible. The structure on top of the backscatterling spectrum Indicates that this film is not uniform. It is Ge rich near the surface. At the 
high energy edge the slope of the spectrum is determined by the detector resolution. At the Interface between fllm and substrate the slope is considerably less steep. This is due to straggling In the energy loss. Energy straggling becomes Increasingly Important with Increasing depth, and it finally limits the resolution of backscattering spectrometry. Energy straggling depends on the energy and on the lon-target comblnations. It was treated theoretically in an early paper by Bohr (I) and by Lindhard et. 1. (17). For en example of recent experimantal determinations using backscattering techniques, see Ref. 26 .

The backscattering yleld, i.e. the height $H$ of the energy distributlons (number of counts per channel) is related to the number density of atoms in a layer $\mathrm{dx}$ near the surface:

$$
H=\text { QOSNdx }
$$

where $Q$ is the number of primary particles arriving on the target, $\Omega$ the solid angle subterded by $=t a$ detector, $N$ the number of target atoms per unit volume, and the cress section averaged over the solid angle $(\sigma=1 / \Omega \rho(d \sigma / d \Omega) d \Omega)$. It has been shown ${ }^{(5)}$ that for protons with energies $>50 \mathrm{keV}$ the Rutherford cross section is valid. In laboratory coordlnates this is ${ }^{(27)}$ : $\quad$ do $=\frac{z_{2} z_{2} e^{2}}{16 t} \cdot f(\theta) d \Omega$

$$
f(\theta)=4\left[\cos \theta+\left\{1-\left(\frac{M_{1}}{M_{2}} \sin \theta\right)^{2}\right\}^{\frac{1}{2}}\right] \sin ^{-4} \theta\left\{1-\left(\frac{M_{2}}{M_{2}} \sin \theta\right)^{2}\right\}^{\frac{1}{2}}
$$

where $Z_{1}, Z_{2}$ and $M_{1}, M_{2}$ are the nuclear charges and mass numbers of projectlles and target atoms, e the elementary charge, and $\theta$ the scattering angle. Using formula (2), $d x$ in formula (3) can easily be related to an energy Interval $\mathrm{dE}_{2}$ (for Instance that corresponding to the width of a channel in the multichannel analyzer), when it is assumed that $d E / d x$ is 
constant for the energy interval under consideration. If the functional energy dependence of the stopping cross section from energy is known, formulae for the backscattering yield from thick targets can be derived $(28,29)$. With these the stopping cross sections can be determined from the absolute height of the energy distributions $(28,30,31)$.

If the particles are backscattered from a thin film of thickness $t$, the sum of the counts in all channels containing counts due to particles backscattered from this film is:

$$
\int H\left(E_{2}\right) d E_{2}=\text { QORNt }
$$

This is independent of the stopping powar. Thus the number of target atcms per unit aren, Nt, can be determined directly.

As an example in Fig. 5 the backscattering of 150 keV protons from a Nb film on Be substrate is shown (32). This film was sputtered by $5 \mathrm{keV}$ deuterons. From the decrease of the number of backscattered particies after sputtering the sputtering yieid could be determined.

Beckscattering is a very unlikely process in this energy range. To Illustrate this let us assume a I un thick $\mathrm{Ni}$ foil which is bombarded by 1 MoV protons. A detector of $1 \mathrm{~cm}$ diameter In a distance of $10 \mathrm{~cm}$ frem the target at $\theta=135^{\circ}$ counts only $1.1 \times 10^{-7}$ particles per incident lon. Nevertheless this is a very useful and nondestructive method for surface analysis. In some cases surface impurities of less than $10^{-4}$ monelayers have been detected (33).

\section{MEDIUM ENERGIES}

As mentloned al ready in the introduction, surface barrier detectors are no longer suitable to measure particle energies below $20 \mathrm{keV}$. It is. 
stralghtforward in this energy range to use electrostatic or magnetic spectrometers. These are, naturally, only sensitive to charged particles. There are several papers $(4,34-37)$ which report on measured energy distributions of the charged component of the backscattered particles. Most of the backscattered particles are, however, neutral at energies below 40 keV (for hydrogen) $(10,11,12)$. A comparison with the theoretical values is only possible if the total number of backscattered particles is known since the theory of the charged fraction is not yet well developed. Therefore, a direct measurement of the neutrals seemed to be desirable. Buck et. 1. (13) successfully performed time of flight measurements for He in the energy range of 6 to $32 \mathrm{keV}$.

Another method, which we have used at the IPP in Garching, is to ionize the neutral particles by stripping in a gas cetl $(10,11)$. The principle is explained by Fig. 6. A magnetically selected ion bean impinged onto the target, which could be rotated such that the entrance and exit angles $\alpha$ and $\beta$ could be varied. A scattered beam intensity corresponding to a scattering angle of $\theta=135^{\circ}$ was selected. With deflection plates the charged component could be removed from the beam. In the stripping cell, which was filled with $2 \times 10^{-3}$ :Torr $M_{2}$, ia part (known from a previous calibration) of the neutrals was ionized and energy analyzed in a $90^{\circ}$ electrostatic spectrometer which ut ilized a channeltion multiplier detector.

Representative measurements of the energy distributlons of positively charged and neutral particles backscattered at $\theta=135^{\circ}$ from a Ta target bombarded with $18.5 \mathrm{keV}$ protons are shown in Fig. 7. The charged fraction $Q=N^{+} / N^{+}+N^{0}$, i.e. the number of positively charged to the number of 
neutrals plus positives, decreases from $\approx 40 \%$ at $20 \mathrm{keV}$ to $\approx 10 \%$ at 2 keV. Both the neutral and the charged spectra show distinct maxima at low energles. It is also seen that besides their relative helght, the shapes of the two spectra are rather different. Consequently, a derivatilon of the neutral spectrum from the charged is not easily accompiished. The shapes of the backscattering spectra depend only slightly on the target moterial. For decreasing primary energy the maximum is more pronounced, but the charged fraction depends only on the exit energy. The charged fraction decreases slightly with increasing angle of emergence $B^{(11)}$. At low energies negative particles were also observed (38). In this case the negative fraction is smil, but especially with target materials with low work function, the negative fraction may be much larger than the positive.

In separate experiment an electrostatic analyzer was used, which could be swivelled around the target in order to determine the angular distribution of the partlcles backscattered when proton bean is $1 \mathrm{~m}^{-i}$;: pinging normal onto a Nb target ${ }^{(39)}$. With this instrumentation only the charged component (including positive and negative ions) could be masured. For protons on Nb it was possible to determine the total number of backscattered particles using the charged fraction measured previously (11).

It is shown in Ref. 39 that the angular distribution of all backscattered particles is very close to a cosine distribution for primary protonienergies from 4 to $15 \mathrm{keV}$. Only the ions scattered with the highest energies are preferrential scattered into smaller scattering angles. These contribute, however, only very little to the total back- 
scattering intensity. Two examples of energy distributions of all particles backscattered into the whole half-space are shown in Fig. 8. Both spectra with primary energies $E_{2}=10.22 \mathrm{keV}$ and $E_{2}=4.16 \mathrm{keV}$ (measured with $8.32 \mathrm{keV} \mathrm{H}_{2}+$ ions) show a distinct maximum at $\approx 1 \mathrm{keV}$. This is due to the fact that the scattering cross section increases as the lons lose energy in the solid. Below the maximum the probability of multiple scattering and removal from the beam exceeds the probability of backscattering events. By integration of the spectra in Fig. 8 over all energies the reflection coefficient $R$, i.e. the number of backscattered to Incoming particles, can be determined. In Fig. 9 our results on the backscattering coefficients are shown together with theoretical and experimental values from other authors. $R$ increases rapidly as the primary energy is lowered. In the theoretical work of Weissmann and Sigmund $(40)$ and of B $\phi$ ttiger and Winterbon ${ }^{(41)}$ the slowing down of the protons in an amorphous solid of infinite extent is calculated by means of the Boltzmann transport equation. This model assumes that the atoms start from a plane in the solid. All atoms which finally come to rest behind this plane are considered to be backscattered. Bфttiger and Winterbon ${ }^{(41)}$ also include a surface correction. The values of J. E. Robinson ${ }^{(42)}, 0,5$. Oen and $M$. T. Robinson (43) as well as the value for Mo by ishitani, et. al. (44) were obtained by computer simulation. These simulations also assume amorphous materials.

The fact that the experimental results are considerably lower than those from the calculations can probably be attributed to two main differences: (1) the Nb target had rather large crystal grains. Thus the backscattering was more from individual single crystals than from amor- 
phous material. This leads to larger penetration depths and hence less backscattering. For $E_{1}=10 \mathrm{keV}$, Den ${ }^{(43)}$ also simulated a polycrystalline target and obtained a backscattering coefficient, which is much smaller than the value for amorphous material. (2) The $\mathrm{Nb}$ was very likely covered with an oxide layer which also reduces the backscattering.

In Fig. 9 recent experimental results of sidenius ${ }^{(45)}$ measured on Au are included. (To compare the values for Au with those for Nb they were plotted at reduced energies ${ }^{(17)} \varepsilon=E \cdot$ a $M_{2} /\left[\left(M_{1}+M_{2}\right) Z_{2} Z_{2} e^{2}\right]$, where $a=0.468\left(z_{1}{ }^{2}+z_{2}{ }^{3}\right)^{-\frac{1}{2}} \AA$ is the screening length of the interaction potential.) Sidenius had his target mounted inside a proportional counter so that all the backscattered particles were absorbed and created pulses proportional to their energy. These resu?ts are close to the theoretical values. An oxide layer cannot be expected in the case of Au, which indicates again the influence of such a layer on our results.

Recently Bфttiger and Rud ${ }^{(46)}$ determined the trapping coefficient of $\mathrm{He}^{3}$ in Au using a nuclear reaction. When no gas is released thermally, the sum of the trapping and reflection coefficients is unity. The values obtained by this method are also in good agreement with theory.

The knowledge of reflection coefficients as well as energy and angular distributions is very important for plasma experiments. Therefore, more experiments are necessary. Another important number in this context is the energy reflection coefficient $\gamma$, i.e. the total energy carried away by the backscattered particles related to the incoming energy. It is $\gamma=R \bar{E} / E_{3}$, where $\bar{E}$ is the mean energy of all reflected particles. $\bar{E}$ can be determined from the spectra as in Fig. 8. The energy reflection coefficient $\gamma$ was recently directly measured by a Danish group ${ }^{(47)}$ using 
different calorimetic methods. They find good agreement with the computer simulation of O. S. Oen and M. T. Robinson (43).

\section{LOW ENERGIES}

At very low energies the fraction of the backscattered particles which are charged becomes very small. At energies below $\approx 200 \mathrm{eV}$, the ionization method by stripping in a gas cell breaks down and because the cross sections for electron loss are small compared to those for scattering in the gas and the latter effect distorts energy and angular distributions. In the energy range above several tens of eV the ionization by electron impact is also impossible since the required electron densities cannot be achieved. The detection methods for neutrals also break down. All currently used methods for detecting neutrals depend on the creation of secondary electrons. At energies where the potential emission of ions dominates the kinetic emission ( $\leqslant 200 \mathrm{eV}$ ) there is no: longer any emission of secondary electrons by neutrals. Because of the lack of detectors also the time of flight methods are then no longer usable at these low energies.

Therefore, one has to rely on the results of computer simulations. These are, in turn, especially suitable for low energies since it is easy to obtain sufficient stạtistics without too much computer time. Energy and angular distributions for protons scattered from Cu obtained recently by 0 . S. Den and M. T. Robinson ${ }^{(43)}$ are shown in Fig. 10. The energy distribution with a primary energy of $5 \mathrm{keV}$ shows a maximum at $\approx 1 \mathrm{keV}$ which agrees with the experimental observations. At a primary energy of $100 \mathrm{eV}$ the spectrum is sharply peaked at high energies corresponding to 
backscattering from the surface. The angular distributions show remarkable deviations from a cosine distribution, which would give the dotted lines. The backscattered intensity is peaked in the entrance di rection which was normal to the surface. At grazing incidence the authors found reflection coefficients close to 1 with the intensity highiy peaked in the direction of specular reflectance.

\section{ACKNOWLEOGMENTS}

I am indebted to B. R. Appleton, J. W. Miller, O. S. Oen, M. T. Robinson, H. H. Anderson, and J. Bфttiger for snaking their results available to me prior to publication. I appreciate a critical reading of the manuscript by B. R. Appleton and O. S. Den. Finally I want to thank the people of the Solid State Division of ORNL for their warm hospitality 1 experienced during my year in Oak Ridge. 


\section{REFERENCES}

1. N. Bohr, Kgl. Dan. Vid. Selsk. Mat. Fys. Medd. 18, No. 8 (1948).

2. J. Linclhard, V. Nielsen : and M. SScharff;, Kgi . Dan. Vid. Selisk: Mat. Fys. Medd. 36, No. 10 (1968).

3. S. Rubin, Nucl. Inst. Meth. 5, 177 (1959).

4. G. H. McCracken and N. J. Freeman, J. Phys. B 2, 661 (1969).

5. A. van Wijngaarden, E. J. Brimmer and W. E. Baylis, Can. J. Phys. 48, 1835 (1970).

6. E. Rutherford, Phil. Mag. 21, 669 (1911).

7. W. F. Hornyak, T. Lauritzen, P. Morrison and W. A. Fowler, Revs. Mod. Phys. 22, 291 (1950).

8. F. K. McGowan, W. T. Milner, H. J. Kim and Wanda Hyatt, Nuclear Data Tables 6, 353 (1969); Z, 1 (1969).

9. See for instance: "Ion Beam Surface Layer Analysis" ed. by J. W. Meyer and J. F. Ziegler, Elsevier. Sequoia, Lausanne (1974).

10. R. Behrisch, W. Eckstein, P. Meischner, B.M.U. Scherzer and H. Verbeek, Atomic Collisions in Solids, ed. by S. Datz, B. R. Appleton, and C. D. Moak, Plenum Press, N.Y. 315 (1975).

11. P. Meischner and H. Verbeek, J. Nucl, Mat. 53, 276 (1974) and Report IPP, 9/18 (1975).

12. T. M. Buck, L. C. Feldman and G. H. Wheatley, Atomic Collitisions in Solids, ed. by S. Datz, B. R. Appleton and C. D. Moak, Plenum Press, N.Y. 331 (1975). 
13. T. M. Buck, Y. S. Chen, G. H. Wheatley and W. F. van der Weg, Surface Sclence $47,244-255$ (1975).

14. E. Hinnov, J. Nucl. Mat. 53, 9 (1974).

15. D. S. Gemmel 1, Revs. Mod. Phys. 46, 129 (1974).

16. D. V. Morgan (ed.), "Channeling, Theory, Observation and Applications", Wiley, N.Y. (1973).

17. J. Lindhard, M. Scharff and H. E. Schiфtt, Kgl. Dan. Vid. Selsk. Mat. Fys. Medd. 33, No. 1.4 (i963).

18. W. Whaling, "Encyclopedia of Physics", ed. by S. Flïgge, Springer Ver1. 34, $193(1958)$

19. L. C. Northcliffe and R. F. Schilling, Nucl. Data Tables A Z, 233 (1970)

20. J. F. Ziegler and W. K. Chu, Atomic Data and Nucl. Data Tables 13 , No. $5(1974)$.

21. P. Sigmund, Proceedings of the Advanced Study Institute on Radiation Damage Processes in Materials, Corsica (1973).

22. W. H. Bragg and R. Kleeman, Phil. Mag. 10, S 318 (1905).

23. J. S.-Y. Feng, W. K. Chu and M.-A. Nicolet, Thin Solid Films 19, 227 (1973).

24. D. K. Brice, Thin Solid Films 19. 121-135 (1973).

25. J. W. Miller, B. R. Appleton and J. R. Gavaler to be presented at the International Conference on Ion Beam Surface Layer Analysis, Karlsruhe, Sept. 15-19 (1975).

26. J. M. Harris, W. K. Chu and M.-A. Nicolet, Thin Solid Films 19, 259 (1973). 
27. For a derivation of this formula, see J. F. Ziegler and R. F. Lever, Thin Solid films 19, 291 (1973).

28. R. Behrisch and B. M. U. Scherzer, Thin Solld Films 19, 247 (1973).

29. W. K. Chu and J. F. Ziegler, J. Appl. Phys. 46, 2768 (1975).

30. W. A. Wenzel and W. Whaling, Phys. Rev. 87, 499 (1952).

31. D. Powers and W. Whaling, Phys. Rev. 126, 61 (1962).

32. W. Eckstein, B. M. U. Scherzer and H. Verbeek, Rad. Effects 18, 135 (1973).

33. J. D. Ball, T. M. Buck, C. W. Caldwell, D. McNair and G. H. Wheatley, Surf. Sci. 30, 69 (1972).

34. E. R. Cawthron, D. L. Cotterel and M. Oliphant, Proc. Roy. Soc. Lond. A319, 435 (1970).

35. K. Morita, H. Akimune and T. Suita, Jap. J. Appl. Phys. Z, 916 (1968).

36. W. Eckstein and H. Verbeek, J. Vac. Sci. Technol. 9, 612 (1972).

37. E. S. Mashkova and V. A. Molchano\%, Rad. Effects 13, 131 (1972).

38. H. Verbeek, W. Eckstein and S. Datz, to be published.

39. H. Verbeek, J. Appl. Phys. 46, 2981 (1975).

40. R. Weissmann and P. Sigmund, Rad. Effects 19, 7 (1973).

41. J. Bqttiger and K. B. Winterbon, Rad. Effects 20, 65 (1973).

42. J. E. Robinson, Rad. Effects 23, 29 (1974).

43. O. S. Oen and M. T. Robinson, to be presianted at the 6th International Conference on Atomic Collisions in Sollds, Amsterdam, Sept. 22-26 (1975).

44. T. Ishitari, R. Shimizu and K. Murata, Jap. J. Appl. Phys. 11, 125 (1972). 
5. G. SIdenius, Phys. Lett. A 49,409 (1974).

6. J. Bфttiger and $N$. Rud, to be presented at the International Conference on the Application of Ion Beams to Materials, September 8-12, 1975, Warwlck, England.

7. H. H. Anderson, T. Lenskjaer, G. Sidenlus and H. S\$rensen, to be pubiished in J. Appl. Phys. 


\section{FIGURE CAPTIONS}

Fig. 1. Energy distribution of hydrogen atoms backscattered from Au which is bombarded by $15 \mathrm{keV}$ protons.

Fig. 2. Principle of the backscattering of light ions from a solid.

Fig. 3. Differential energy loss for $H$ and He ions in $M I$ from Ref. 19.

Fig. 4. Backscattering spectrum of $2 \mathrm{MeV}$ He from a $\mathrm{Nb}_{3} \mathrm{Ge}$ film on an $\mathrm{Al}_{2} \mathrm{O}_{3}$ substrate ${ }^{(25)}$.

Fig. 5. Energy distributions of protons backscattered at an angle of $\theta=135^{\circ}$ from a $600 \AA \mathrm{Nb}$ film on a substrate before and after sputtering with $5 \mathrm{keV} \mathrm{D}^{+}$ions $(32)$.

Fig. 6. Experimantal setup for the detection of neutral backscattered particles.

Fig. 7. Energy distributions of noutral and positively charged hydrogen etoms beckscattered from To bomberded with 18.5 keV protons. The charged fraction $N^{+} / N^{+}+N^{\circ}$ is given by dots.

Fig. 8. Energy distributions of all partleles backscattered into $2 \pi$ solid angle when a $\mathrm{Wb}$ target is bombarded with $10.22 \mathrm{keV} \mathrm{H}_{1}^{+}$ and $8.32 \mathrm{koV} \mathrm{H}_{2}{ }^{+}$iens.

Fig. 9. Exporimentel and theoretical reflection coefficlents for hydrogen as a function of the primery energy.

Fig. 10. Energy (left) and angular (right) distrubutions of hydrogan atcoms becksentered from Cu bommarded with 100 oV and 5000 of protons. (Computer simulation by an and Robinson (43) .) 


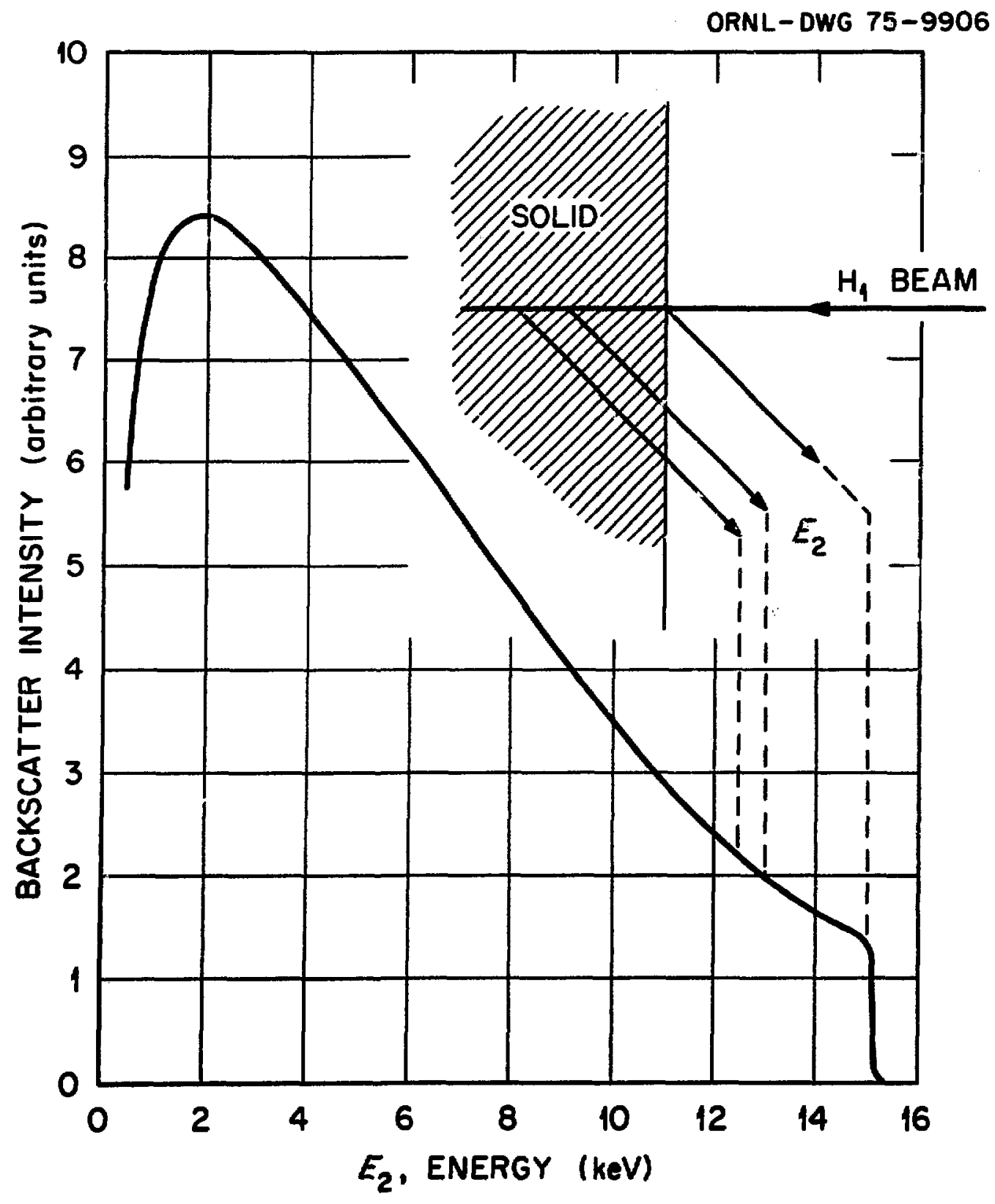

Fig. 1 


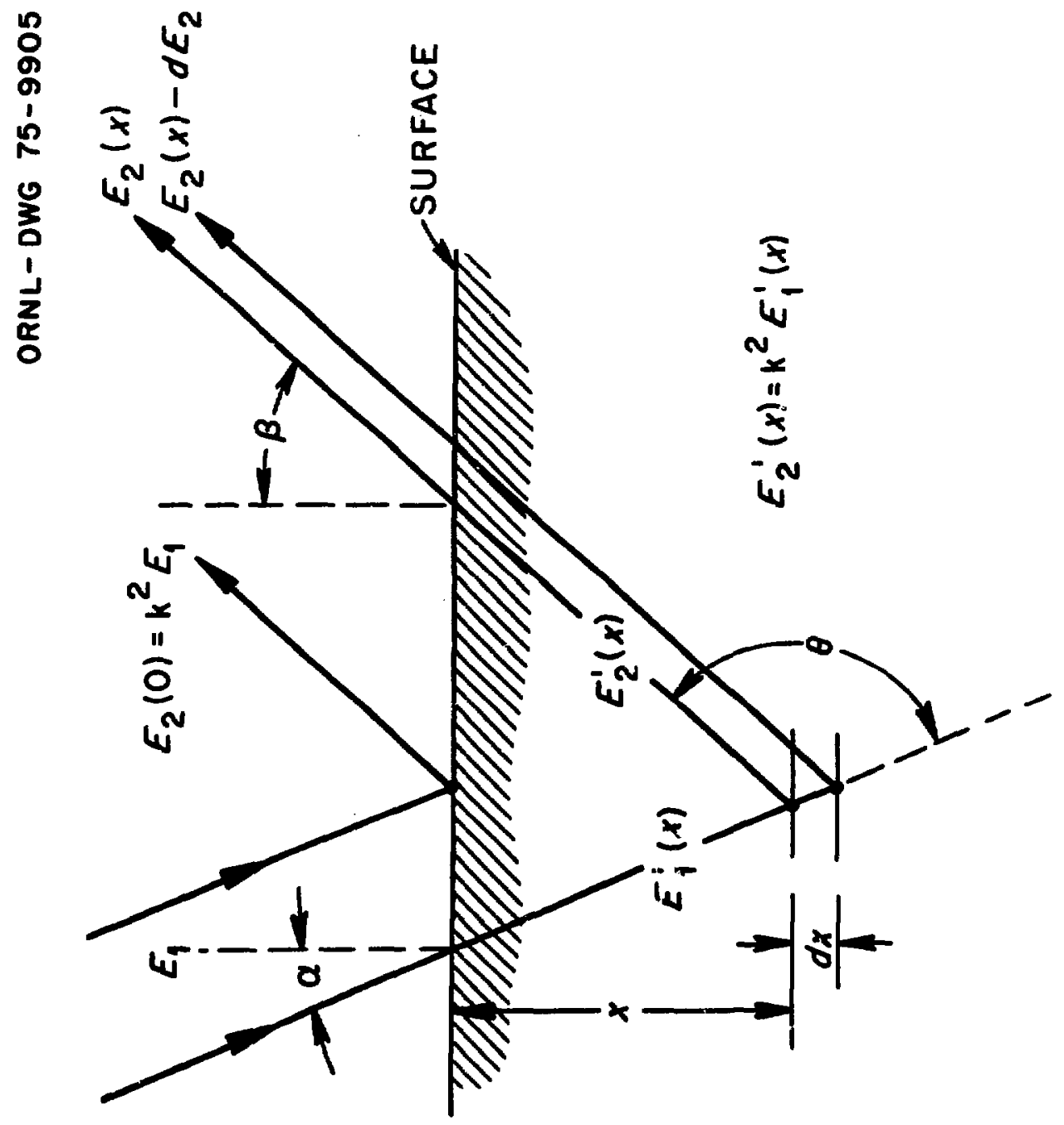

iv 


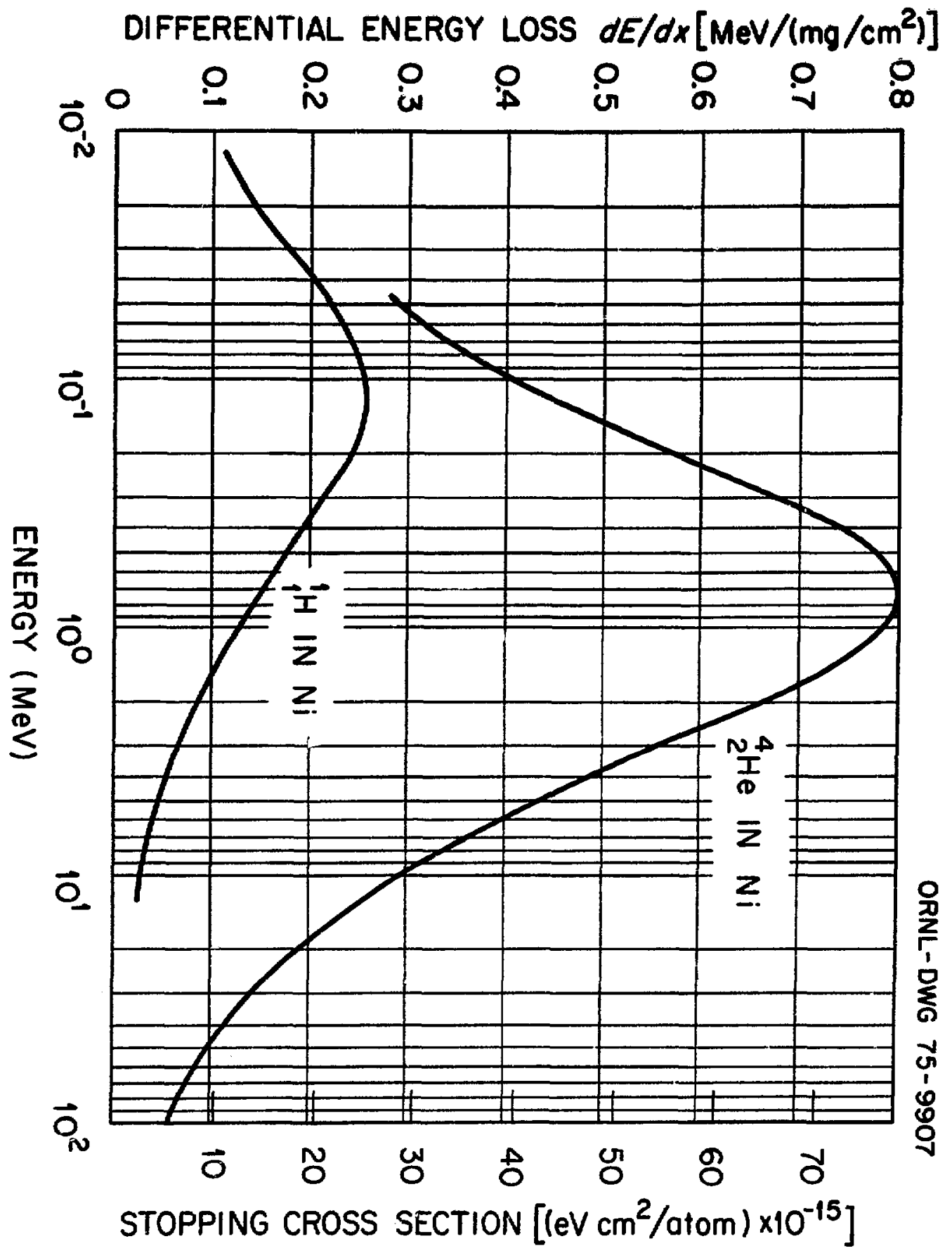




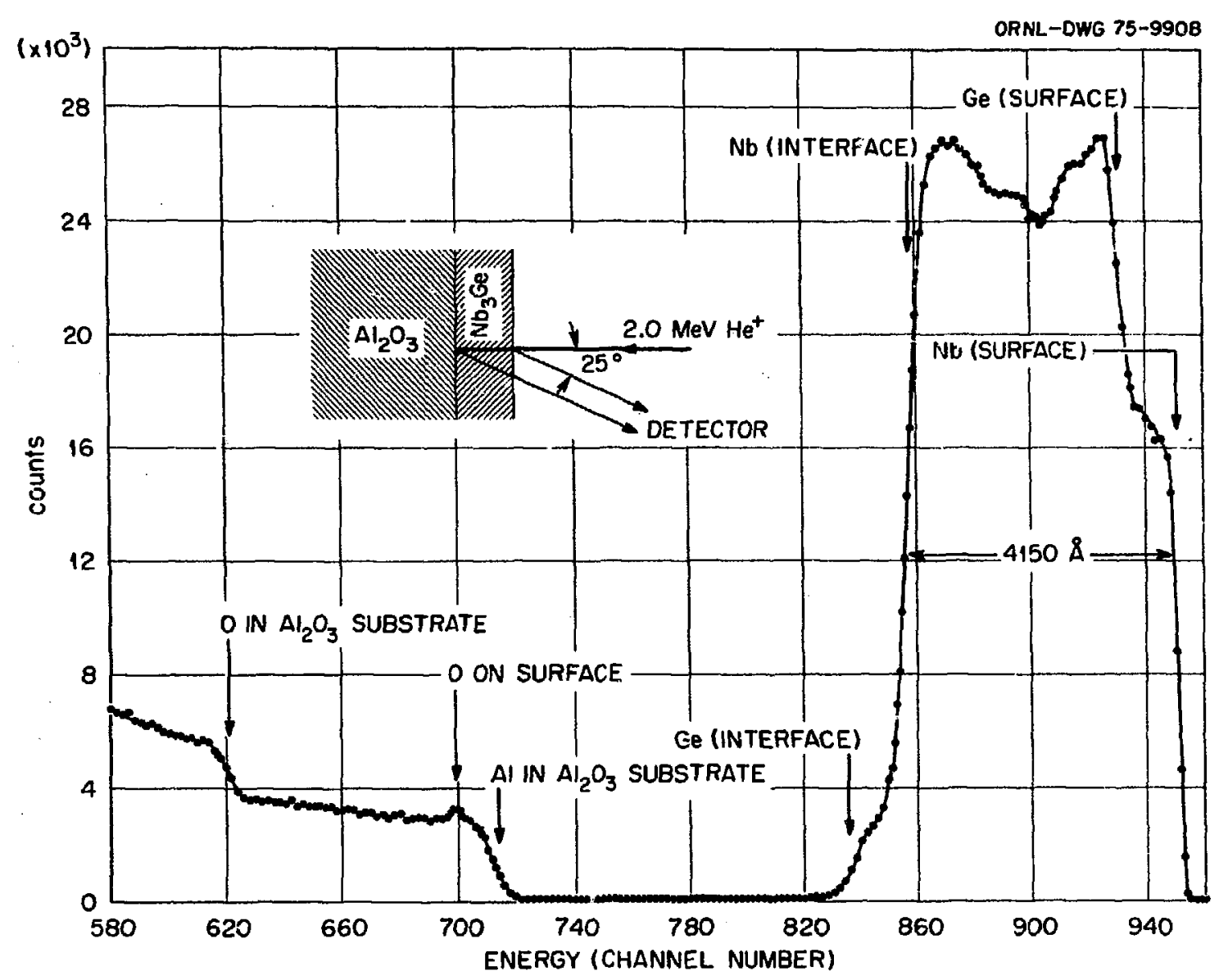

Fig. 4 

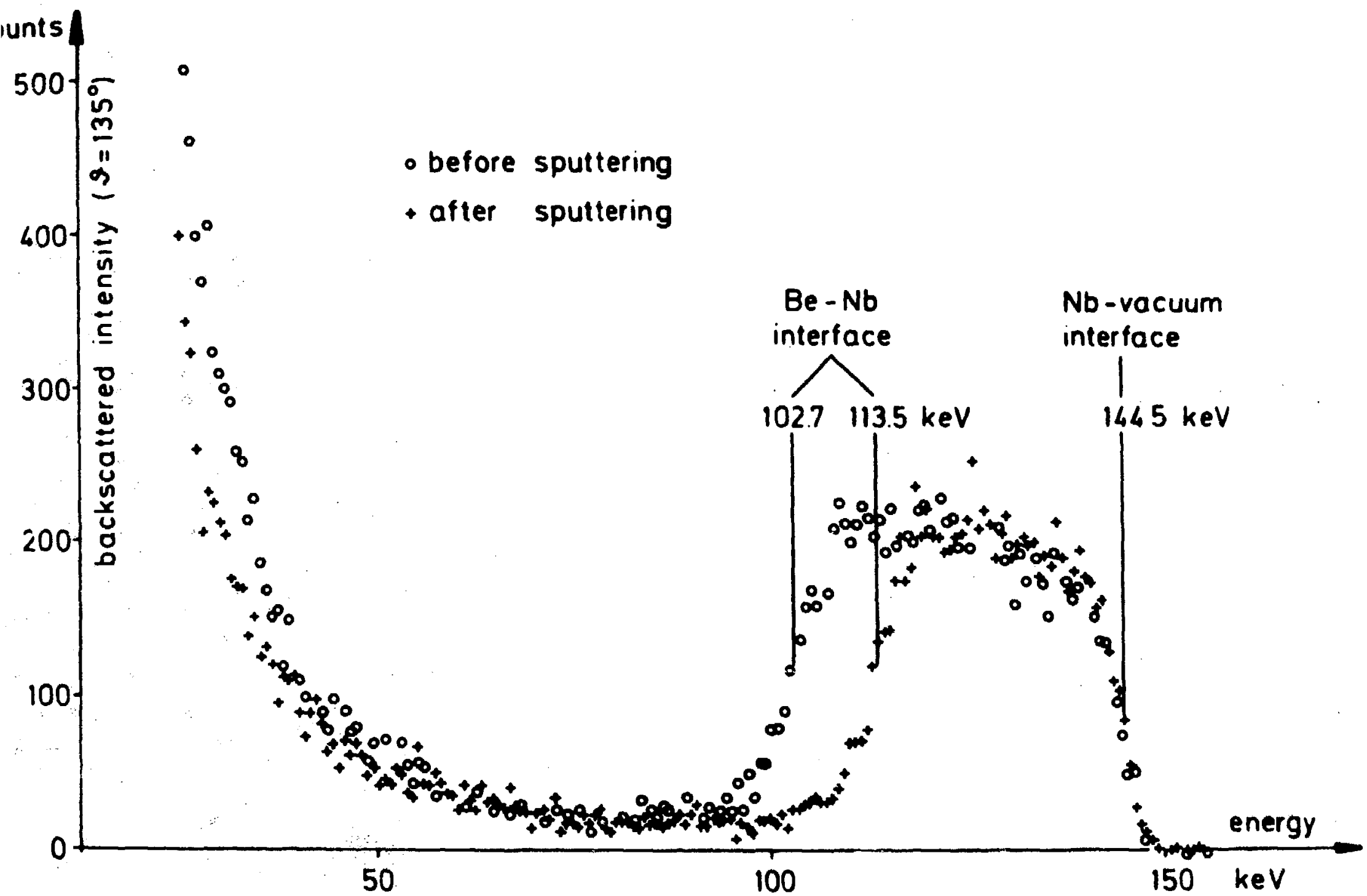

Fig. 5 


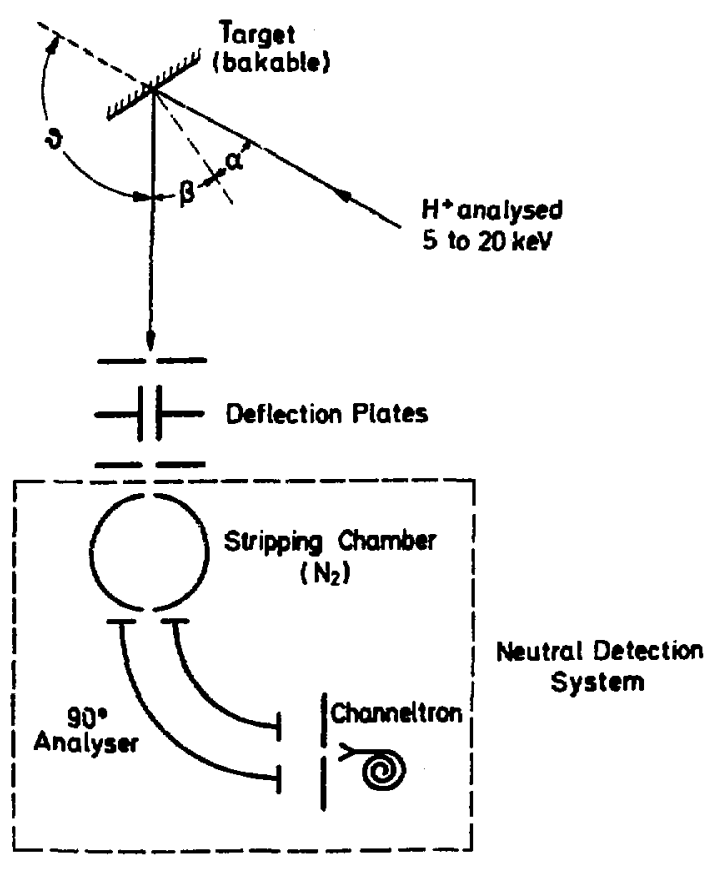

fig. 6 
PARTICLES PER ENERGY INTERVAL larb. units)

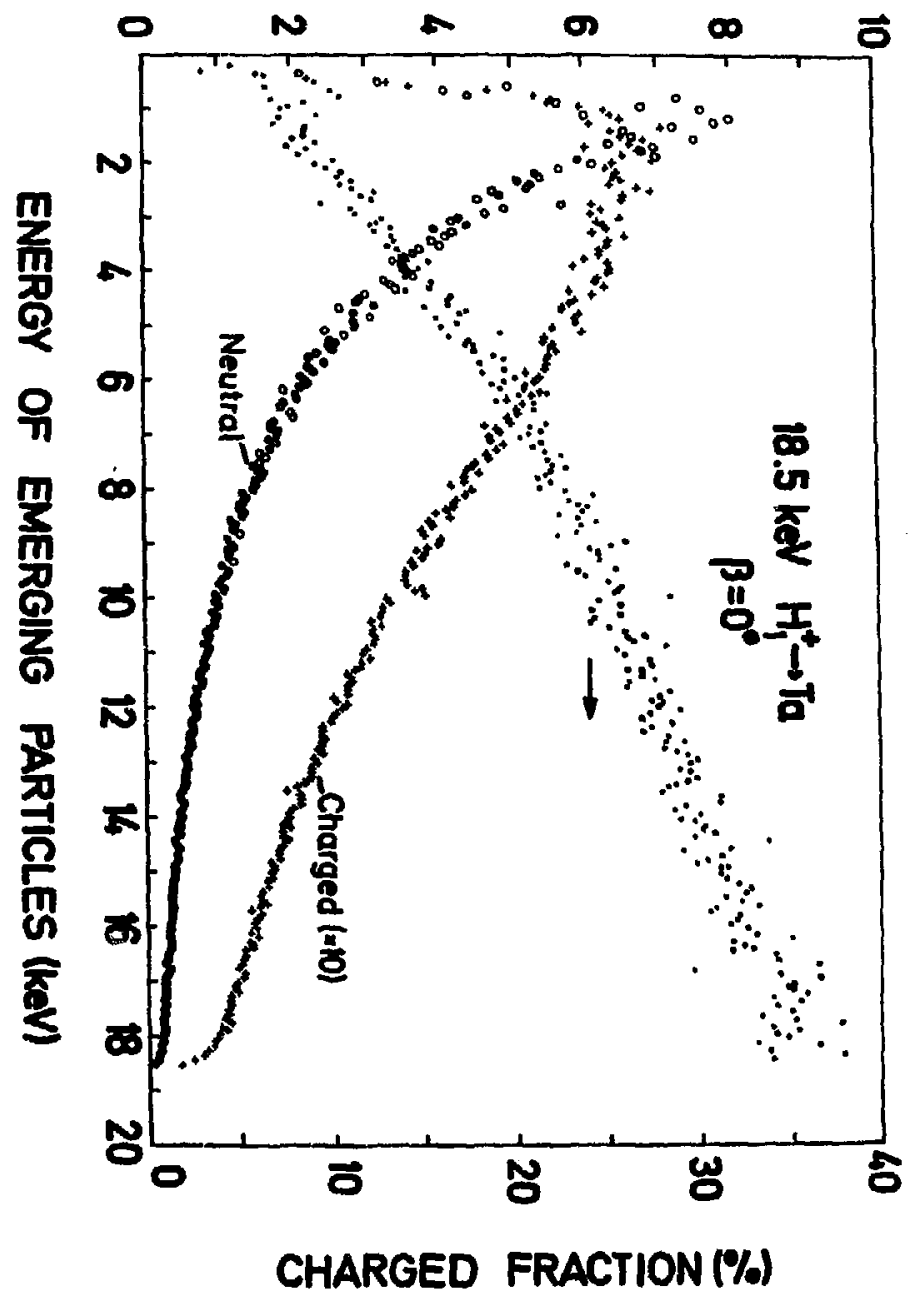




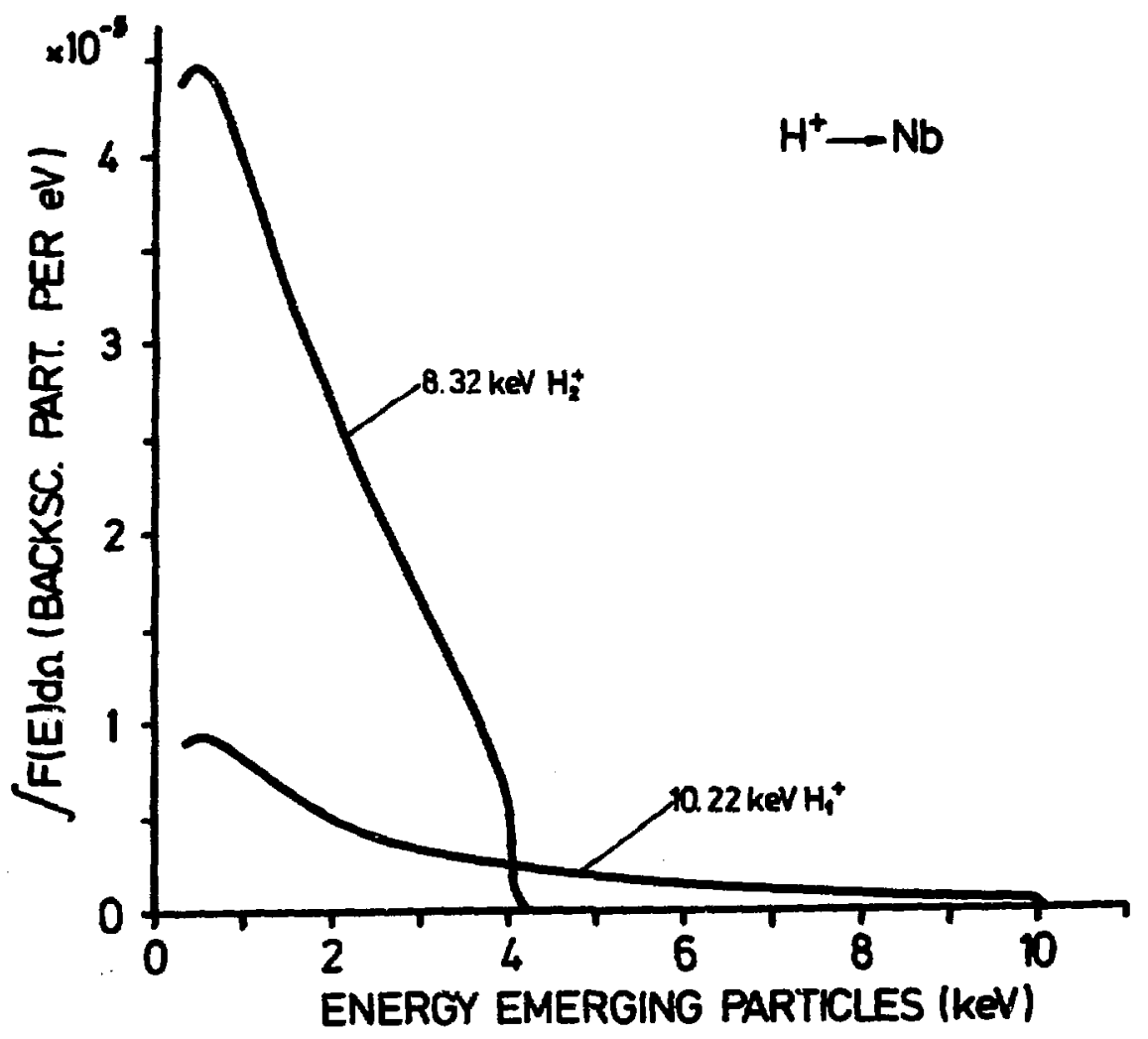

Fig. 8 


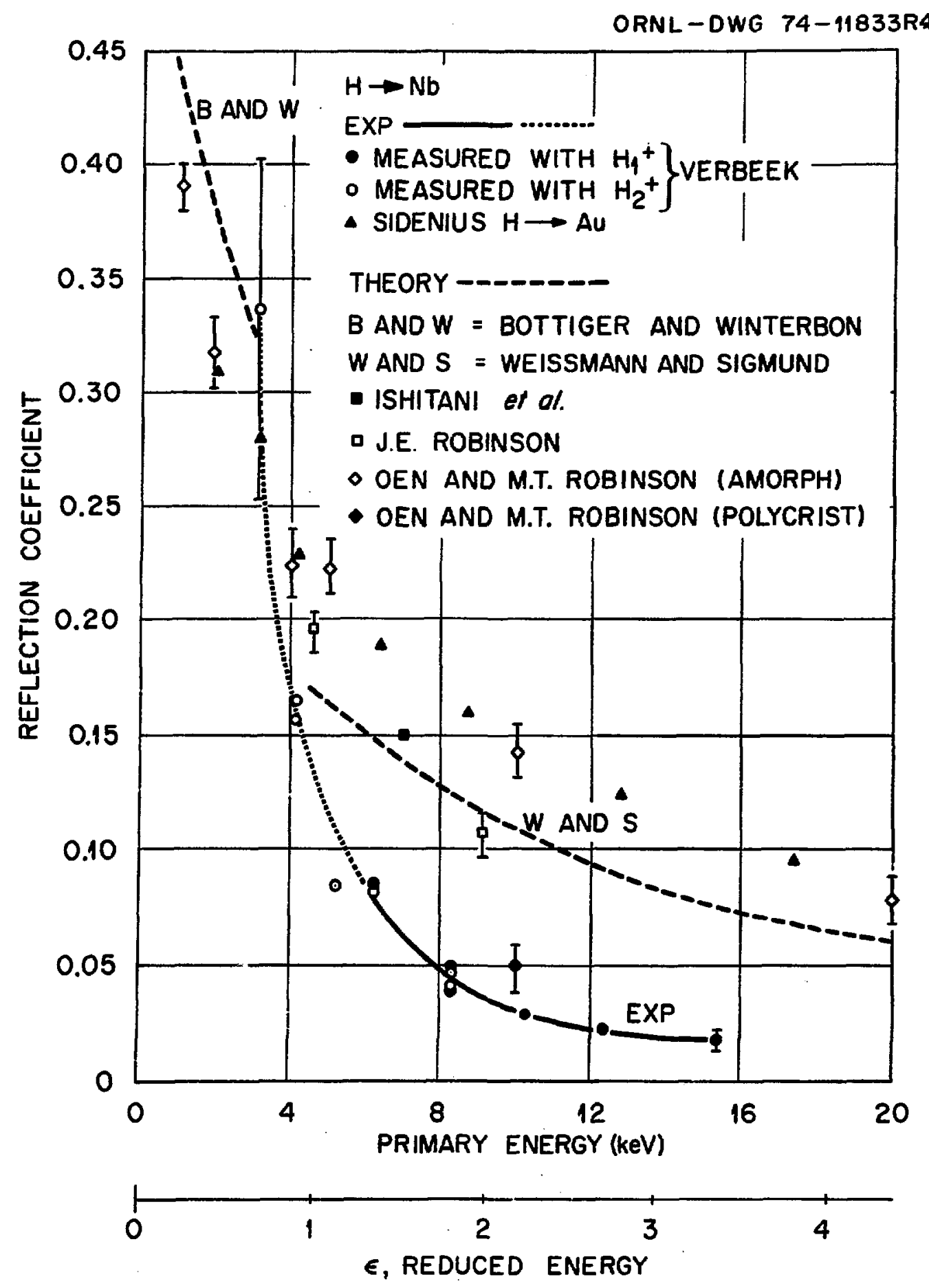

Fig. 9 
OnNL-OW6 75-30H

POLAR REFLECTION ANGLE (dag)
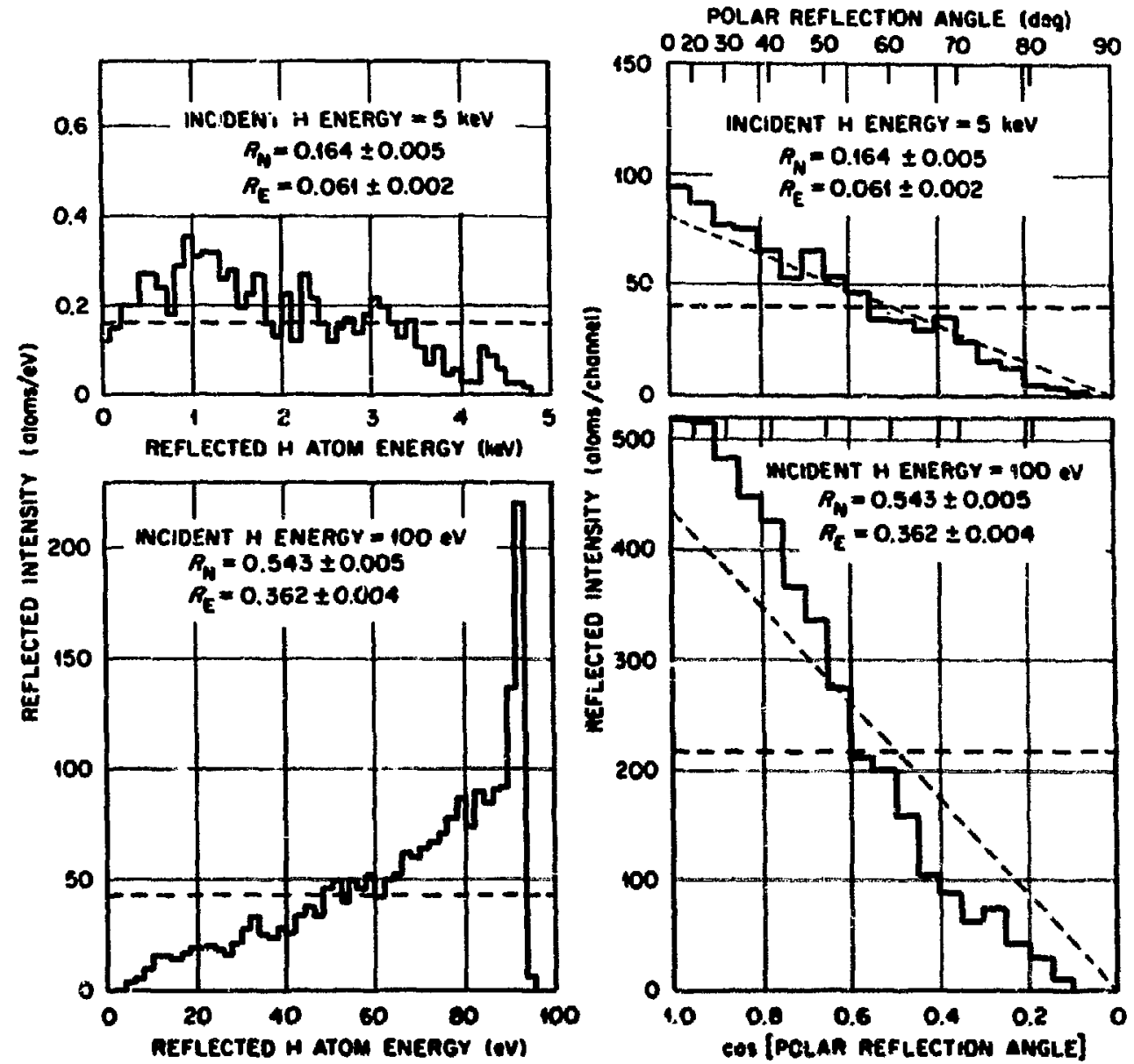

Ratiection of $\mathrm{H}$ from Amorphous Copper. 